\title{
Relating Properties and Functions from Patents to TRIZ Trends
}

\author{
P.-A. Verhaegen ${ }^{1}$, J. D'hondt ${ }^{1}$, J. Vertommen ${ }^{1}$, S. Dewulf ${ }^{2}$, J. R. Duflou ${ }^{1}$ \\ ${ }^{1}$ Centre for Industrial Management, Dept. Mechanical Engineering, K.U.Leuven, Belgium \\ ${ }^{2}$ CREAX N.V., leper, Belgium
}

\begin{abstract}
TRIZ trends describe the evolutionary status of a system by identifying the trend phases, and assist in predicting improvements by identifying evolutionary potential. This process encompasses analyzing and categorizing patents in known trend phases, relying on intrinsic skills of a TRIZ expert, and depicting the results on an evolutionary potential radar plot. To structure this approach, an algorithm is proposed that, through patent analysis and identification of word categories, extracts information concerning the product properties, which relate to trend phases. Allowing controlled and repeatable measurements of trends, this algorithm can support the problem specification and requirements gathering phases.
\end{abstract}

\section{Keywords:}

Technology Forecasting, TRIZ Trends, Patent Analysis

\section{INTRODUCTION}

\subsection{Technological Forecasting}

Technology Forecasting (TF) indicates all processes to anticipate the generic or specific direction of technological change of a product or family of products, primarily focusing on inventions and innovations. Generally, TF has four roles towards steering R\&D and technology related decisions on a managerial level [1]. However, for the research underlying this paper a more restricted definition of TF is used, limiting its role to monitoring specific technology and planning for technology development.

Besides the well-known and widely used trend extrapolation and Delphi methods, there exists a multitude of other TF methods, which can be categorized as quantitative versus qualitative, and as normative (goaloriented) versus exploratory (extrapolation of current technological capabilities)[1] [2]. TRIZ trend analysis can be categorized as being quantitative, as it is based on the observations of metrics, such as the number of patents. Although TRIZ trends are certainly among the more formalized and quantitative methods, it must be noted that the gathering of the underlying metrics relies on TRIZ expert skills.

\subsection{TRIZ}

TRIZ is the Russian acronym for the Theory of Inventive Problem Solving, and encompasses a series of tools and a methodology for generating innovative ideas and solutions for problem solving. It was formed through the systematic analysis of what TRIZ practitioners estimate to be one and a half to three million patents, in which the applied innovative solutions were mapped onto a small number of extracted inventive principles.

TRIZ is based on three postulates [3] [4]:

- The Postulate of Existing Objective Laws states that engineering systems evolve according to a set of laws

- The Postulate of Contradictions states that, in order to evolve, an engineering system has to overcome one or more contradictions.
- The Postulate of the Specific Situation states that the problem solving process should take into account the specific problem peculiarities.

Derived from this patent analysis and based on the postulates, a set of TRIZ tools was conceived, of which the most popular are [5]:

- The Contradiction Matrix to solve technical contradictions;

- The Separations Principles to solve physical contradictions;

- Substance-Field (SU-Field) modeling and the Inventive Standards to transform technical systems;

- ARIZ as a list of logical procedures for eliminating contradictions;

- TRIZ Trends as a system of laws that govern engineering system evolution.

It should be noted that, in contradiction to the other TRIZ tools, TRIZ trends are almost solely based on the Postulate of Existing Objective Laws. Some of the other tools are also based on this postulate, and trends can be easily identified in these tools, e.g. the Inventive Standards - Class 2: Evolution of Systems [4] [5].

Several examples of the integration of TRIZ in the conceptual and embodiment stages of the classical design process exist [6]. In combination with Quality Function Deployment (QFD), TRIZ allows to focus efforts on the relevant mechanism identified through analysis of the customer's needs [7][8]. Other literature has illustrated the complementarity of TRIZ with Axiomatic Design [9][10] and Robust Design [11], and the integration of TRIZ with DFMA, resulting in a methodology in which DFMA analysis is used to evaluate TRIZ generated alternative solutions [12].

\subsection{TRIZ Trends}

Classical TRIZ identifies eight laws of development of systems, subdivided in static, kinematic and dynamic laws [4]. The three static laws enumerate the necessary criteria for the viability of technical systems, and describe 
which parts a system must contain, and how the periodicity of operation of these parts must be coordinated or purposely uncoordinated and that these parts must freely conduct energy through the system. The kinematic laws govern the evolution of technical systems regardless of system specific conditions. These laws state that, as a system evolves, it increases its degree of idealness, and that development of the system's parts occurs unevenly, resulting in new technical and physical contradictions. The third kinematic law expresses that, when a system exhausts its evolutionary potential, it is incorporated in its super-system as one of its parts, and as a result it can further evolve. The more system-specific dynamic laws indicate the tendency of the working units to act on a smaller scale, and also point towards increasing (electromagnetic) S-Field involvement. A hierarchical overview of these laws can be found in [13], which divides these laws into laws of system organization and laws of system evolution. Some literature also explicitly states the Scurve law, as the cycle of birth, growth, maturity, and decline which every technical system goes through [14]. The above laws are in fact hypotheses, and Savransky [5] (p.96) proposes to name them trends because they were obtained through induction of patterns in patents, and no formal proof has been given yet. As the majority of literature refers to these hypotheses as laws, and to the lines of evolution as trends, this article uses this nomenclature.

A number of these laws include more specific sequences of transitions or trends, indicating how a system or its parts evolve over time [13]. An updated, non-hierarchical list of thirty-five trends, incorporating new domains and reflecting new innovative solutions, can be found in [15]. These more specific trends should be regarded as the evolution of general properties of the parts of the system. This research focuses on these updated trends as this allows for a more actual and specific categorization, and this is considered more practical in predicting future improvements of a technical system through evolutionary potential. This concept of evolutionary potential can be defined as the difference between the limits of each of the evolution trends and the relative maturity of the current system with respect to these trends [16].

The following section gives an overview of the related research on technological maturity, evolutionary potential, patent analysis and the integration of TRIZ in the design process. The third section describes the proposed methodology, while the fourth illustrates this methodology with a case study. The final section formulates the conclusions.

\section{RELATED RESEARCH}

The relationship between the technological maturity, or the lifeline of technological systems, and the four metrics proposed by Altshuller [4] was validated on a case study basis in [17][18] [19]. To circumvent difficult to measure metrics, such as the profitability or the performance metric, these studies often use indicators, such as the number of patents that used the technology, or don't take these metrics into account. Besides Altshuller's four metrics, [18] also reviews the number of cost reduction related inventions and the number of symptom-curing related inventions as technology maturity metrics. This research determines the overall-maturity of a product family on an S-curve, but does not indicate which trends have more evolutionary potential. It is therefore less specific as input to the planning for technology development.
Other research by Cavallucci [6] proposed and validated the possibility to incorporate the eight original Altshuller's laws of development in the design process on a manifold case study. For each of the eight laws, the product under consideration was positioned on a zero to three scale, a process heavily relying on the expert's intrinsic TRIZ skills. Based on TRIZ and domain knowledge, the conclusions concerning the development potential can be translated into specific directions for future improvements of the manifold. It was also concluded that the difficulties a company faces when integrating this approach, are mainly related to the building up of TRIZ competence and the time and money required to do this.

Building on Cavallucci's research, the proposed approach addresses these difficulties by automating the process of positioning the product on the trends. Furthermore, by using updated and more specific trends, the interpretation of the evolutionary potential and the drawing of conclusions concerning possible improvements is facilitated.

In [20] and [16], Mann and Dewulf propose the concept of evolutionary potential, which is similar to the approach proposed by Cavalucci, but uses the more specific trends, allowing for a more actual and specific categorization.

As stated in the introduction, TRIZ is based on the results of manually analyzing millions of patents. To actualize this information, automatic patent analysis and classification systems can be used. However, these systems are based on technology-dependent schemes, such as the International Patent Classification (IPC), and fail to satisfy TRIZ user requirements. TRIZ users are more interested in analogous inventions in other fields that solve the same contradiction using the same generic inventive principles. [21] proposes an automatic patent classification to categorize patents in six selected inventive principle classes, and also evaluates the performance of different clustering algorithms on this task. [22] proposes a text-based expert system for TRIZbased patent classification according to Inventive Principles, which are grouped in 22 new classes based on distinctiveness, and text and meaning similarity. Other methodologies allow to identify the architecture of the invention by identifying the components, their compositional relationship, and functional interaction [23][24].

\section{PROPOSED METHODOLOGY}

To further formalize and quantify the process to obtain the evolutionary potential, this research proposes an algorithm and framework that, through patent analysis and identification of adjectives, can extract information concerning the properties of a given product or product family, which in turn can be related to particular trends or trend phases. Allowing for more controlled and repeatable measurements of TRIZ trends, this algorithm can be incorporated in the product design specification phase to support the design engineer during problem specification and requirements gathering.

To identify the evolutionary potential of a product family, the available patents dedicated to the product are subjected to analysis. Identifying the trend phases for each of those patents, and positioning these on the trend dimensions in a radar plot gives an overall view of the product family. This radar plot provides valuable input to technological forecasting based on these TRIZ trends.

This analysis uses updated specific trends [15], which capture the evolution of a property of the product. For the scope of this article, we define a property as "what a product is or has", it's attributes. This is mainly expressed 
in adjectives and is related to physical parameters [25]. Examples of properties are hollow, smooth, transparent, strong, and flexible. These are all generic, in contrast to product-specific attributes, for example light-weight or inspectable. These product-specific attributes are related to functional requirements [25]. A generic property such as hollow can lead to a product-specific property as lightweight, just as transparent can lead to inspectable.

Mann gives an overview and explanation of thirty-five trends [15]. These trends are sequences of trend phases, of which two examples are provided below. Most products follow the trends from left to right, but TRIZ theory encompasses the possibility of reversal under recent circumstances.

- Space Segmentation trend:

Monolithic solid -> Hollow -> Multiple hollows -> Capillary / porous -> Porous with active elements

Examples: Bricks, chocolate bars, tires.

- Dynamization trend:

Immobile -> Jointed -> Fully flexible -> Fluid or

pneumatic $->$ Field-based

Examples: Steering, doors, chairs.

Typically, in determining the evolutionary potential, only a certain number of most frequently identified trends is taken into account. This allows to focus on the most important trends and to not encumber the interpretation of the evolutionary potential radar plot.

Patents related to a specific product or product family can be gathered using a combination of International Patent Classification (IPC) or other categorization codes, and text search facilities on the different sections of the patents. Other possibilities encompass the related patents section, or matching on inventors, or applicants.

The retained patents are then subjected to Part-OfSpeech (POS) tagging to identify the adjectives. Some patent sections contain specific numerical or textual information, such as patent number, date of application and authors. Other, more narrative, patent sections are:

- The title of the invention

- The abstract

- The claims section

- The background section

- The summary section

- The description section

[21] indicates the importance of including the titles and abstracts in the automatic classification of patents, while the summary section gives only marginal improvements. Other research shows that the inclusion of a certain number of words or lines of the description, applications and/or claims can be beneficial to patent classification [26] [27]. In the proposed approach, only the title and the abstract are tagged, although the other sections will be analyzed at a later stage. An additional benefit is that for most patents, the title and the abstract are available in English, which is not always the case for the other patent sections.

Currently several modules of a test platform have been implemented, of which some have been graphically depicted in Figure 1. The patents are structured XML-files, which are fed into a XSLT transformation module. Through this transformation the text in the title and abstract fields is concatenated and retained for further processing. Next a tokenizer module splits the text, as a string of characters, into a set of tokens, which can be interpreted by a POS-tagger.

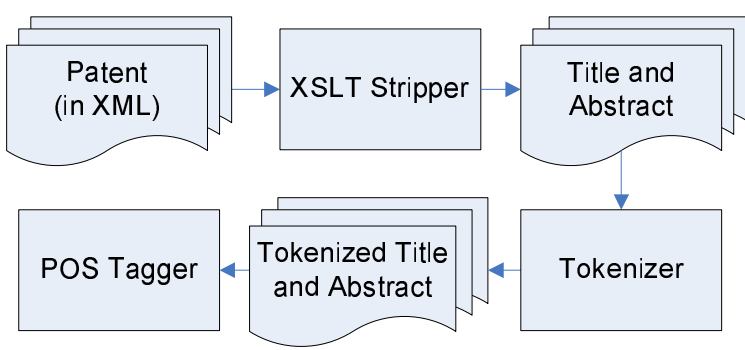

Figure 1 : Currently implemented modules of the test platform

The tagger allows to identify the adjectives, which are used as an aid to identify the relevant TRIZ trends and trend phases in the patents. Currently, this trend and trend phase identification step is performed manually, but further research aims at automating this task.

The subsequent phases in a trend are represented by integers, ranging from one to the number of phases for the trend. Since the various trends have a different number of trend phases, the identified trend phase is normalized with respect to the total number of phases in that trend. Consequently, the maximum and average normalized trend phases are calculated for each trend and graphically depicted on a radar plot. To simplify the interpretation of this evolutionary potential chart, the trends are grouped according to time, space and interface categories [15].

\section{CASE STUDY}

To illustrate the proposed methodology, a set of 5900 patents related to the umbrella product family was retrieved through the IPC code A45B. As this set also covers walking sticks and the like, only patents including the word umbrella in the title were withheld. From this, twelve patents were randomly selected, of which the title and abstract were Part-Of-Speech (POS) tagged using a non-trained TnT-Tagger [28]. The results of the POS tagging are the identified adjectives depicted in Table 1.

A number of non-adjective words were incorrectly categorized by the TnT-Tagger as adjectives. Visa versa, some adjectives are not recognized as such. Based on these twelve patents, the best estimate is that fifteen percent of the identified adjectives belong to other word categories, while twelve percent of the adjectives were not identified as such. Currently, a TnT-Tagger is being trained on a corpus of randomly picked patents, the outcome of which will be compared to the current results. Trying to eliminate manual intervention, the proposed adjectives have not been manually corrected.

A number of words were incorrectly identified as adjectives, e.g. patent number EP1354533A3. This occurs when the tagger encounters constructions such as "loudspeaker system", "textile cover", "volume control", or "earphone jack". Being nouns that modify other nouns, these words are in fact attributive nouns or noun adjuncts [29]. It should be noted that [30] describes a method "Keyword and phrase extracting" which allows for the identification of multi-words phrases, based on the assumption that these multi-words would occur several times in the document. However, in the proposed research, trends are studied as the evolution of properties of the product, and the case study indicates that these noun modifiers are indicative of the evolution of the system, and beneficiary to the trend phase categorization. Further research will evaluate the usefulness of incorporating other word categories in the trend phase identification. 
In Table 1, the underlined adjectives, are used as an aid to manually identify the trends and trend phases. For most patents, the adjectives give a clear indication of the relevant trend, for example for patent US6840253B2 the adjectives movable, pivotal and rotatable represent the trend dynamization. However, for some patents, such as patent US6158451A, the retained adjectives give little to no indication of the trends. Further research aims at identifying the causes and possible solutions, such as including other word categories, or analyzing more patent sections. For these patents, the relevant phases within each trend were identified manually, a task which future research aims at automating.

After determination of the most likely trend phase(s) for each patent, these were normalized to account for the different number of phases in trends. Consequently, the maximum and average normalized trend phase was calculated for each trend. As the precise patent distribution along each trend is difficult to obtain on such a small data set, the average and maximum metrics are to be interpreted with care, and only give an indication of the distribution and evolutionary potential. In a later stage of this research, automatic identification of trends based on adjectives will be investigated, as this would allow for reducing human involvement and processing larger patent sets.

The average and maximum trend phase metrics are visualized in Figure 2, allowing for easy assessment of the evolutionary potential of the umbrella product family. In this figure, the 'Controllability', 'Reducing Human Involvement' and 'Increasing Use of Senses' are related to the TRIZ Interface concept and have been grouped together. For the same reason all other trends are grouped as they are related to the TRIZ Space concept, except the 'Mono-Bi-Poly (various)', which is the only relevant trend related to the TRIZ Time concept [15].
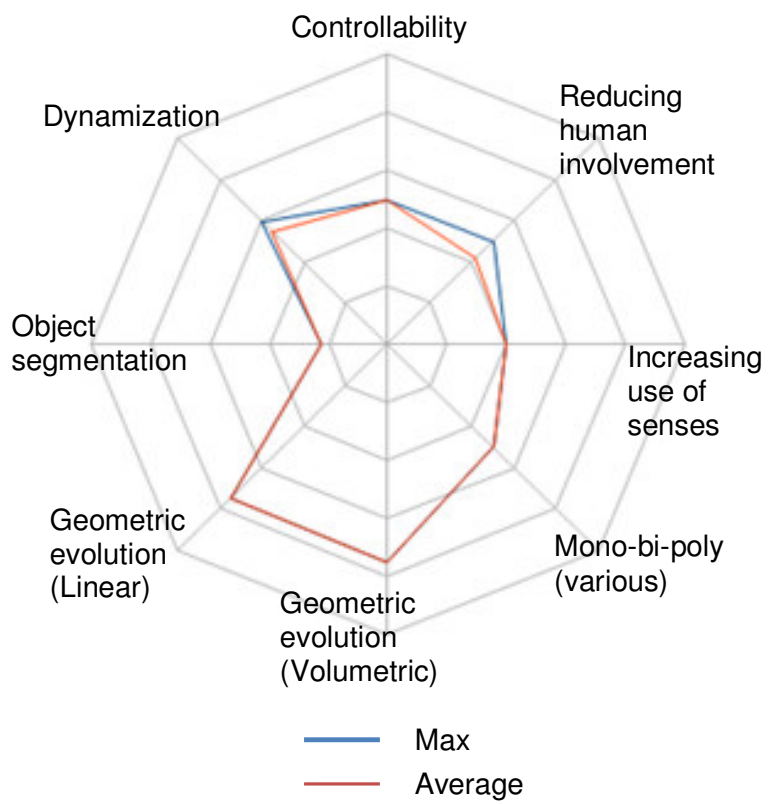

Figure 2: Evolutionary Potential of the umbrella product family

The evolutionary potential radar plot indicates that margins for further improvements to the umbrella product family exist, especially along the segmentation and the "increase use of senses" trend. Although this research does not aim at identifying specific improvements, one could, for instance, imagine the use of a fabric changing color depending on the dry or wet state of the fabric, an improvement which is to the best of our knowledge not yet available or patented.

The evolutionary potential radar plot does not include all relevant trends for the umbrella product family, and would benefit from increasing the number of analyzed patents. Analyzing more patents also leads to a more solid basis as input to technology monitoring and planning for technology development.

\section{SUMMARY}

By means of a case study of the evolutionary potential of the umbrella product family, it was shown that analyzing the adjectives of the patents related to the umbrella product family gives an indication of the trends for each patent. These trends and trend phases are the basis for discovering the evolutionary potential of the umbrella product family. Further research aims at automating this task and the identification of the phases within these trends to provide a more solid basis for technology monitoring and planning for technology development.

Allowing for more controlled and repeatable measurements of the evolutionary potential according to TRIZ trends, this algorithm can be incorporated in the product design specification phase to support the design engineer during problem specification and requirements gathering.

\section{REFERENCES}

[1] Yoon, B., Park, Y., 2007, Development of New Technology Forecasting Algorithm: Hybrid Approach for Morphology Analysis and Conjoint Analysis of Patent Information, IEEE Transactions on Engineering Management, 54/3:588-599.

[2] Porter, A.L., et al., 2004, Technology Futures Analysis: Toward Integration of the Field and new Methods, Technological Forecasting and Social Change, 71/3:287-303.

[3] Khomenko, N., Ashtiani, M., 2007, Classical TRIZ and OTSM as a Scientific Theoretical Background for Non-Typical Problem Solving Instruments, Proceedings of the TRIZ-Future Conference, 73-80.

[4] Altschuller, G S., 1984, Creativity as an Exact Science - The Theory of the Solution of Inventive Problems, Gordon and Breach Science Publishers, New York.

[5] Savransky, S. D., 2000, Engineering of Creativity: Introduction to TRIZ Methodology of Inventive Problem Solving, Boca Raton, Florida.

[6] Cavallucci, D., 2002, Integrating Altshuller's Development Laws for Technical Systems into the Design Process, Annals of the CIRP, 50/1:115-120.

[7] León-Rovira, N., 2002, A proposal to integrate TRIZ into the product design process, The TRIZ Journal.

[8] YamaShina, H., Ito ,T., Kawada, H., 2000, Innovative Product Development Process by Integrating QFD with TRIZ, Journal of the Japan Society of Precision Engineering, 66/1:1705-1710.

[9] Yang, K., Zhang, H., 2000, A Comparison of TRIZ and Axiomatic Design, Proceedings of ICAD2000 First International Conference on Axiomatic Design, Cambridge, MA, 235-242.

[10] Duflou, J., Dewulf W., 2006, On the complementarity of TRIZ and Axiomatic Design: From decoupling objective to contradiction 
identification. Proceedings of the TRIZ future Conference, Kortrijk, Belgium.

[11] Hu, M., Yang, K., Taguchi S., 2000, Enhancing Robust Design with the Aid of TRIZ and Axiomatic Design (Part I), The TRIZ Journal.

[12] Lucchetta, G., Bariani, P., 2005, Integrated Design Analysis for Product Simplification, Annals of the CIRP, 54/1:147-150.

[13] Petrov, V., 2002, The Laws of System Evolution, The TRIZ Journal.

[14] Dung, P., 2001, Enlarging TRIZ and Teaching Enlarged TRIZ for the Large Public (Part 1 of 4), The TRIZ Journal.

[15] Mann, D. L., 2002, Hands on Systematic Innovation, CREAX Press, leper, Belgium.

[16] Mann, D., Dewulf, S., 2002, Evolutionary-Potential in Technical and Business Systems, The TRIZ Journal.

[17] Gibson, N., 1999, The Determination of the Technological Maturity of Ultrasonic Welding, The TRIZ Journal.

[18] Mann, D., 1999, Using S-Curves and Trends of Evolution in R\&D Strategy Planning, TRIZ Journal.

[19] Slocum, M., 1999, Technology Maturity Using Scurve Descriptors, The TRIZ Journal.

[20] Mann, D., 2002, Better Technology Forecasting using Systematic Innovation Methods, Technological Forecasting and Social Change, 70/8:779-95.

[21] Loh H. T., He, C., Shen, L., 2006, Automatic classification of patent documents for TRIZ users, World Patent Information, 28/1:6-13.

[22] He, C., Loh H. T., 2008, Grouping of TRIZ Inventive Principles to facilitate automatic patent classification. Expert Systems with Applications, 34/1:788-795.
[23] Cascini, G., Russo, D., Zini, M., 2007, ComputerAided Patent Analysis: Finding Invention Peculiarities, Monterrey Nuevo León, Mexico.

[24] Cascini, G., Russo, D., 2007, Computer-Aided Analysis of Patents and Search for TRIZ Contradictions, International Journal of Product Development, 4/1-2:52-67.

[25] Dewulf, S., 2006, Directed Variation: Variation of Properties for New or Improved Function Product DNA, a Base for 'Connect and Develop', Proceedings of the ETRIA TRIZ Future Conference, Kortrijk, Belgium.

[26] Larkey L.S., 1999, A patent search and classification system, Proceedings of the fourth ACM conference on Digital libraries, ACM Press, New York, US., 179-187.

[27] Fall, C J, et al., 2003, Automated Categorization in the International Patent Classification, SIGIR Forum, ACM Press, 37/1:10-25.

[28] Brants, T., 2000, TnT - A Statistical Part-Of-Speech Tagger, Proceedings of the Sixth Applied Natural Language Processing Conference ANLP-2000, Seattle.

[29] Noun Adjunct., Wikipedia, (http://en.wikipedia.org/wiki/Noun_adjunct)

[30] Tseng, Y.-H., Lin, C.-J., Lin, Y.-I., 2007, Text Mining Techniques for Patent Analysis, Information Processing and Management, 43/5:1216-1247.

\begin{tabular}{|c|c|c|}
\hline Patent Number & Adjectives & Trends (Normalized Phase) \\
\hline US20020092550A1 & hollow lower upper winding & $\begin{array}{l}\text { Controllability }(0.5) \\
\text { Reducing human involvement } \\
(0.33)\end{array}$ \\
\hline EP1354533A3 & $\begin{array}{l}\begin{array}{c}\text { conventional earphone } \\
\text { electronic incorporated }\end{array} \\
\text { integral loudspeaker normal } \\
\text { Outside photovoltaic textile } \\
\text { tubular unused }\end{array}$ & $\begin{array}{l}\text { Increasing use of senses - } \\
\text { Auditory }(0.4)\end{array}$ \\
\hline US5349975A & $\begin{array}{l}\text { collapsible cool current } \\
\text { electric flat hot rechargeable } \\
\text { separate } \frac{\text { solar therethrough }}{\text { top }}\end{array}$ & $\begin{array}{l}\text { Increasing use of senses - } \\
\text { Kinaesthetic }(0.4) \\
\text { Mono-bi-poly -Various (0.5) }\end{array}$ \\
\hline JP10192022A & $\begin{array}{l}\text { capable decorative equal } \\
\frac{\text { flexible inner lower main }}{\text { planar simple special }} \\
\text { sufficient uniform }\end{array}$ & $\begin{array}{c}\text { Increasing use of senses - } \\
\text { Visual }(0.4) \\
\text { Geometric evolution - } \\
\text { Volumetric }(0.75)\end{array}$ \\
\hline EP623297A2 & central detachable other & Object segmentation (0.22) \\
\hline US5954417A & $\begin{array}{l}\text { alert circular electrical } \\
\text { nighttime other outer } \\
\text { transparent ultrasonic visual } \\
\text { waterproof }\end{array}$ & $\begin{array}{l}\text { Increasing use of senses - } \\
\qquad \operatorname{Visual}(0.4)\end{array}$ \\
\hline US5141010A & $\begin{array}{l}\text { Automatic axial close hollow } \\
\text { least } \frac{\text { manual open proximate }}{\text { radial threaded }}\end{array}$ & $\begin{array}{l}\text { Controllability }(0.5) \\
\text { Reducing human involvement } \\
(0.5)\end{array}$ \\
\hline
\end{tabular}




\begin{tabular}{|c|c|c|}
\hline US3693643A & $\begin{array}{c}\text { adjacent Collapsible } \\
\text { detelescoped lower movable } \\
\text { relative respective roof- } \\
\text { supporting separate } \\
\text { telescoping upper }\end{array}$ & Dynamization (0.6) \\
\hline US6158451A & $\begin{array}{c}\text { attachable convenient easy } \\
\text { other securable selective top }\end{array}$ & $\begin{array}{c}\text { Geometric evolution - } \\
\text { Volumetric (0.5) }\end{array}$ \\
\hline US6840253B2 & $\begin{array}{c}\text { angular corresponding } \\
\text { different engageable free } \\
\text { helical movable pivotal } \\
\text { rotatable tubular }\end{array}$ & $\begin{array}{c}\text { Geometric evolution - } \\
\text { Volumetric (0.75) }\end{array}$ \\
\hline JP2006021003A & $\begin{array}{c}\text { axial bent closed conventional } \\
\text { inconvenient lower normal }\end{array}$ & $\begin{array}{c}\text { Dynamization (0.4) } \\
\text { Geometric evolution - Linear } \\
\text { (0.75) }\end{array}$ \\
\hline US5025819A & $\begin{array}{c}\text { adjacent canopy flexible lower } \\
\text { repellent sling spring-biased } \\
\text { torso upper }\end{array}$ & Dynamization (0.6) \\
\hline
\end{tabular}

Table 1: Adjectives of POS tagged patents with trends and trend phases. 